\title{
Glass slide-based agarose gel electrophoresis for determining the efficiency of RNA amplification and cyanine dye incorporation during RNA labeling
}

\author{
Alex F. Carvalho ${ }^{1}$, E. Jordão Neves ${ }^{2}$, Roberto Hirata, Jr. ${ }^{3}$, and Luiz F.L. Reis ${ }^{1}$ \\ ${ }^{1}$ Ludwig Institute for Cancer Research and Hospital do Câncer, São Paulo, ${ }^{2}$ Instituto \\ de Matemática e Estatística, Universidade de São Paulo, and ${ }^{3}$ Faculdade SENAC de \\ Ciências Exatas e Tecnologia, São Paulo, Brazil
}

BioTechniques 35:688-692 (October 2003)

Global analysis of gene expression using cDNA microarrays is now a commonly used strategy for defining the repertoire of genes that are expressed in cell lines or tissue samples. The overall quality of a microarray data set is highly dependent on each step of the procedure, from spotting to image acquisition and data extraction. Therefore, every attempt to provide quality control to these various steps will improve the quality of the data set and, considering the costs of hybridization, have a positive impact on the project budget. The abundance and quality of RNA are critical issues, especially when the project involves clinical samples, in which minimally invasive techniques are used to obtain biopsy samples. In this scenario, RNA can be a limiting step, and duplicate hybridizations with dye swap might not be feasible. To overcome this problem, several protocols that describe procedures for RNA amplification are available, and extensive comparative analysis of amplified (aRNA) and nonamplified RNAs has proven the validity of this approach (1-3). Concerning the quality of the RNA, one criterion that seems to be relevant is the ratio of $28 \mathrm{~S} / 18 \mathrm{~S}$ of total RNA, which is an indicator of RNA degradation. Another important issue is the efficiency of the labeling reaction and, particularly in competitive hybridizations using two distinct cDNA samples simultaneously, comparison of the specific activity of each labeled sample is critical to ensure not only equal target mass but also equal fluorescence intensity of each labeled cDNA. Here we describe a simple pro- cedure that allows for the quality control of amplification and cyanine dye labeling of aRNA in a single step. The implementation of such controls can avoid hybridization with targets having low specific activity and, in the case of two-color hybridizations, allows for corrections in targets with differences in their specific activity that otherwise could compromise normalization.

In our experiment, $1.5 \mu \mathrm{g}$ total RNA derived from a disease-free human esophagus obtained from our tumor bank were amplified by a T7-based protocol that was slightly modified from Wang et al. (3). After amplification, $3 \mu \mathrm{g}$ aRNA were labeled with either CyTM3- or Cy5- (Amersham Biosciences, Piscataway, NJ, USA) odCTP using random primer in two independent reactions. For analysis, the samples of labeled cDNA were mixed and fractionated through a $1 \%$ agarose gel that was mounted over a glass slide, and migration was performed along the longer axis of the slides using $1 \times$ TAE $(40$ mM Tris-acetate, 1 mM EDTA, pH 9.0) and $60 \mathrm{~V}$. To cast the gel, 2.5-mm polycarbonate plastic strips are fixed along the edge of one standard microscope glass slide $(75 \times 25 \times 1 \mathrm{~mm})$, which creates the gel box. The gel box is then placed into an electrophoresis apparatus (Horizon ${ }^{\circledR}$ 58; Whatman Biometra, Göttingen, Germany). The multiwell comb (14 teeth, $0.8 \mathrm{~mm}$ thick; Whatman Biometra) is carefully adjusted to the gel box, which is then filled with $1 \%$ agarose/1× TAE. After $15 \mathrm{~min}$, the comb is removed, and the apparatus is filled with $1 \times$ TAE. One microliter of each Cy3- and Cy5-labeled samples (repre- senting 1:34 of final volume) was mixed with $1 \mu \mathrm{L} 3 \times$ DNA loading buffer $(15 \%$ glycerol, $3 \times$ TAE, $0.75 \%$ bromophenol blue) and loaded into each well. The fluorescent size marker was prepared by mixing $1 \mu \mathrm{L}$ DNA ladder, $1 \mu \mathrm{L} 3 \times$ DNA loading buffer, and $1 \mu \mathrm{L} 10 \times$ fluorescent dye (Vistra Green ${ }^{\mathrm{TM}}$; Amersham Biosciences). The electrophoresis unit was covered with aluminum foil to protect the gel from light, and electrophoresis was allowed for $45 \mathrm{~min}$ at $60 \mathrm{~V}$. The slide was then placed into a GeneTACTM2000, which is a charge-coupled device (CCD)-based microarray analyzer (Genomic Solutions, Ann Arbor, MI, USA). The images were acquired with the use of a ScanArray ${ }^{\circledR}$ confocal laser scanner (PerkinElmer Life Sciences, Gaithersburg, MD, USA), and the signal intensity for all spots was obtained using QuantArray ${ }^{\circledR}$ microarray analysis software (PerkinElmer Life Sciences). Figure 1 illustrates data analysis performed with the GT tool, which is available with the software package.

In Figure 1A, we show the composite image of the gel captured by individual channels. From left to right, there are Cy5- and Cy3-labeled samples mixed at proportions of 1:2, $1: 1$, and $2: 1$, the Cy3-labeled alone, and size markers. Figure $1 \mathrm{~B}$ shows the same image, and the boxes used for signal quantification are depicted. Box 4 has the same area as boxes 1 , 2 , and 3 and was used to correct for background. In Figure 1C, we have the total signal intensity for each channel (Cy3 in green and $\mathrm{Cy} 5$ in red) and the respective ratios. The image can be used as a second criterion for the efficiency of amplification, together with the necessary quantification in a spectrophotometer or the necessary quantification of amplified RNA. Visual inspection of the sample profile will indicate whether the size distribution is compatible with the expected linear amplification mRNAs. Although this information can also be obtained with commercial products, our procedure can provide additional critical information; namely, the efficiency of labeling. Figure 1, B and C, shows that it is possible to quantify signal intensity from labeled cDNAs, and the expected ratios from the artificial mixture are virtually identical to the experimentally obtained 


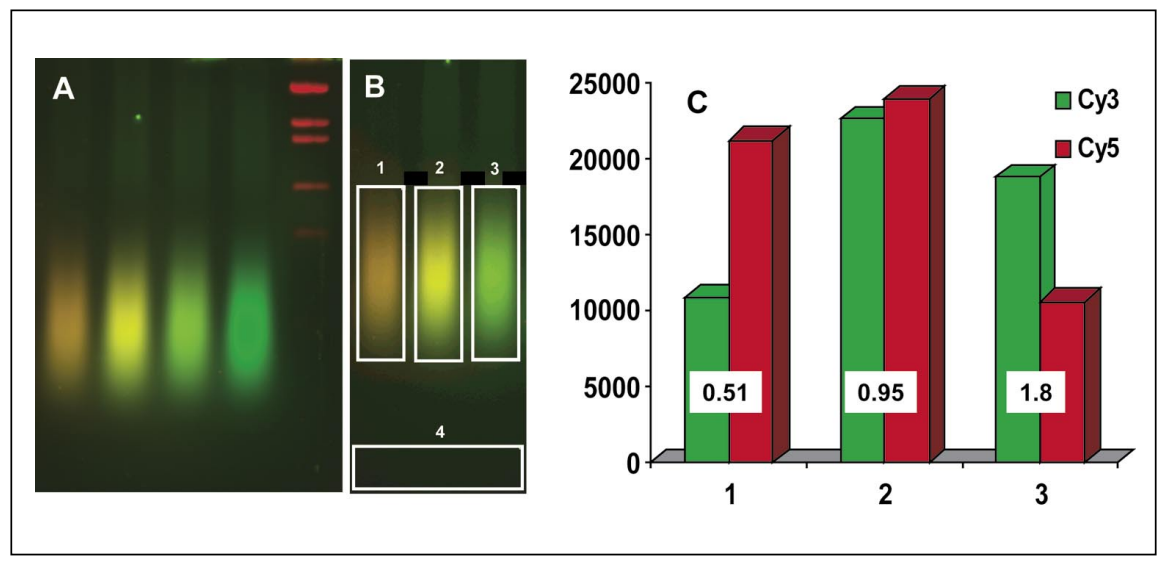

Figure 1. Quantification of labeling efficiency using a mini-agarose gel and a charge-coupled device (CCD)-based microarray analyzer. Total RNA was amplified and labeled with Cy3 or Cy5 by random priming. The samples of labeled cDNAs were fractionated through a $1 \%$ agarose gel, and the image was acquired with a GeneTAC 2000 microarray analyzer. (A) Images of samples mixed (from left to right) at ratios of 1:2,1:1, and 2:1 for $\mathrm{Cy} 3$ and $\mathrm{Cy} 5$, respectively. The forth lane is a Cy3-labeled sample, followed by the size marker. (B) Boxes that were used to calculate labeled efficiency. (C) A histogram showing the total counts for each channel in the three boxes and the corresponding ratio between Cy3- and Cy5-labeled cDNAs.

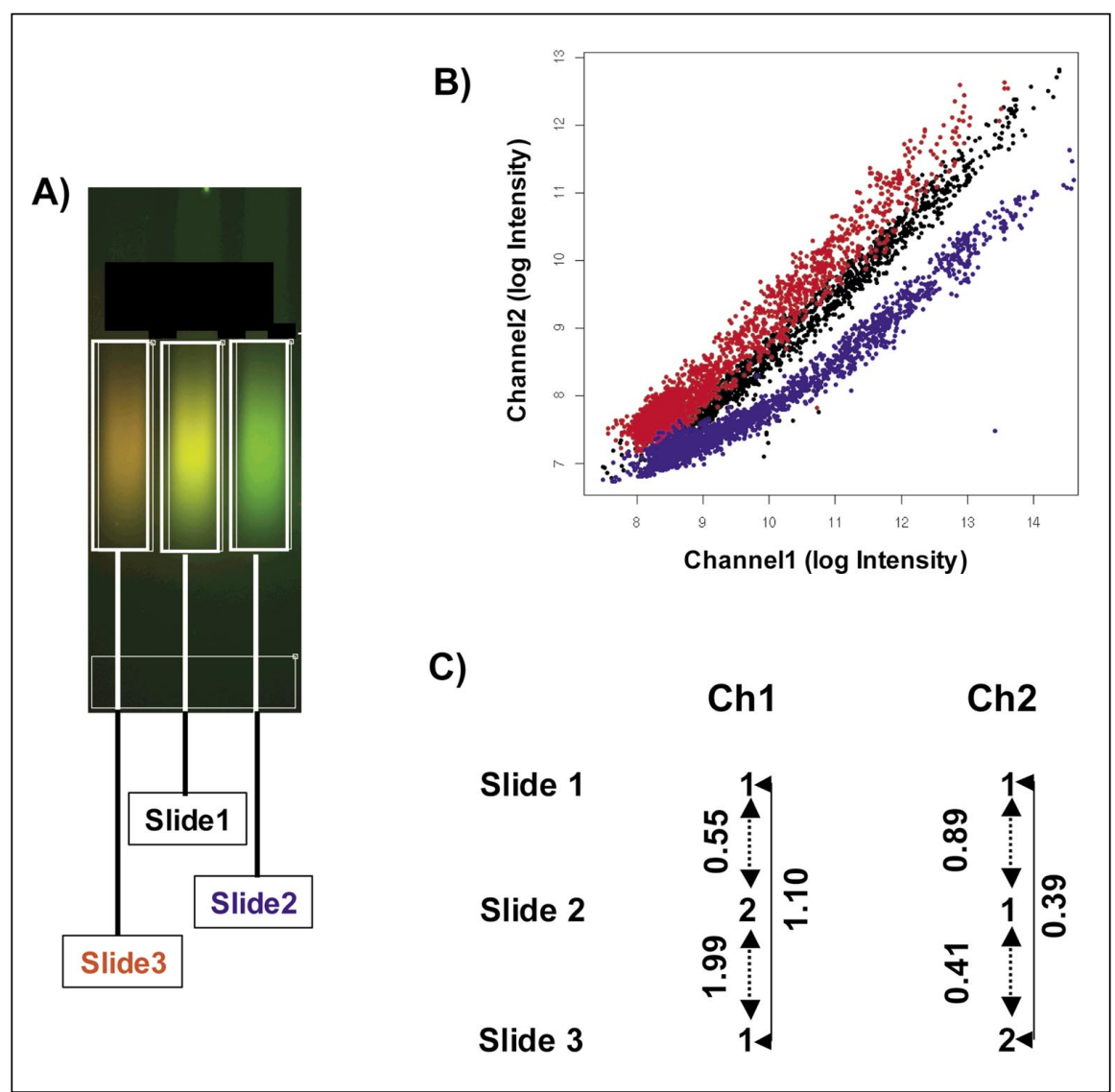

Figure 2. Correlation between intensity ratios determined by scanning of glass slide electrophoresis and by hybridization. (A) Labeled cDNA mixtures were hybridized against glass arrays. An image was acquired using a ScanArray confocal laser scanner, and the signal intensity for all spots was obtained using QuantArray microarray analysis software. (B) A scatter plot of log-transformed signal intensities for both channels of the same slide as indicated in panel A, without any normalization step. (C) The obtained slope regression coefficient value when comparing signal intensities of the same channel for all slides. In the horizontal lines, there are slide numbers and ratios of labeled cDNA mixed for each hybridization. In the vertical lines, there are the coefficient values for the indicated comparisons. ratios. By quantifying signal intensity before hybridization, one can detect undesired discrepancies between the two labeled samples and repeat labeling steps, reducing cost and avoiding data that could impact normalization. To further demonstrate the utility of this procedure, we used the same cDNA mixtures from Figure 1 to hybridize a glass array having 2075 spots and determined the correlation between data from each channel on each slide (Figure 2). After hybridization, the image was acquired on the confocal laser scanner, and the data were extracted using QuantArray software. Data analysis was performed using R (http:// cran.r-project.org/), an open source interpreted computer language for statistical computation and graphics, and tools from the Bioconductor project, adapted to our needs. The Bioconductor project is an international initiative based on $\mathrm{R}$ and dedicated to the development of tools for the analysis and interpretation of genomic data (http:// www.bioconductor.org). We filtered spots for signal higher than background and determined the correlation of data from each channel on all three slides. From the data presented in Figure 1C, one would expect to have three curves with overall ratios of $1: 2,1: 1$, and $2: 1$.

The first step in the analysis of microarray data is the normalization, which is a procedure that is used to compensate for systematic sources of variation $(4,5)$. For slides that are printed on the same batch, as is our case, those procedures usually only involve within-slide normalization, in which effects associated with the dye or printing pin are eliminated. Dye effects are usually quite important, and comparisons of measurements of different dyes often become interpretable only after the appropriate normalization step. However, due to the nature of our experimental design, normalization would mask the expected differences; thus, we plotted the raw data with no normalization steps. Figure 2B shows the scatter plots of Cy5 (channel 2) versus Cy3 (channel 1) measured intensities in log-scale for our three slides, indicated by red (slide 3, 2:1 for Cy5 and Cy3), black (slide 1, 1:1), and blue (slide 2, 1:2 for Cy5 and Cy3). As can be observed, the three sets of data 
points are distributed along lines that represent the expected differences in signal intensity from each channel. Without a systematic dye effect or after proper normalization, we would see three sets of data points along three equally spaced lines parallel with slope one. On the other hand, the direct comparison of measurements for each dye on different slides can be interpreted with no normalization because our experimental setting does not require across-slide normalization. Therefore, the most direct way to assess the efficiency of RNA amplification and Cy3 and Cy 5 dye incorporation during RNA labeling is to directly compare measured quantities for each channel on pairs of slides. Figure $2 \mathrm{C}$ illustrates the results of these comparisons. For each channel, we show the estimated slope for the corresponding intensity comparison, which corresponds precisely to the expected values.

This procedure can be used to monitor the quality of targets after the very last step of the labeling protocol, without compromising the amount of labeled target available for hybridization. Importantly, other commercially available products can efficiently control for amplification but cannot determine the efficiency of the labeling reaction because this would require laser excitation or equivalent wavelengths. Furthermore, one can determine label efficiency using data (Figure 1C) to correct for the specific activity of labeled targets to facilitate data normalization. In the case of greater differences in labeling efficiency, one can repeat the labeling step prior to hybridization, thus avoiding data of lower quality that could impose potential distortions during normalization and eliminating the costs of hybridization.

\section{REFERENCES}

1.Baugh, L.R., A.A. Hill, E.L. Brown, and C.P. Hunter. 2001. Quantitative analysis of mRNA amplification by in vitro transcription. Nucleic Acids Res. 29:E29.

2.Feldman, A.L., N.G. Costouros, E. Wang, M. Qian, F.M. Marincola, H.R. Alexander, and S.K. Libutti. 2002. Advantages of mRNA amplification for microarray analysis. BioTechniques 33:906-914.

3.Wang, E., L.D. Miller, G.A. Ohnmacht, E.T. Liu, and F.M. Marincola. 2000. High-fidel- ity mRNA amplification for gene profiling. Nat. Biotechnol. 18:457-459.

4.Quackenbush, J. 2002. Microarray data normalization and transformation. Nat. Genet. 32(Suppl)496-501.

5.Yang, Y.H., S. Dudoit, P. Luu, D.M. Lin, V. Peng, J. Ngai, and T.P. Speed. 2002. Normalization for cDNA microarray data: a robust composite method addressing single and multiple slide systematic variation. Nucleic Acids Res. 30:E15.
Received 12 May 2003; accepted 8 July 2003.

Address correspondence to Luiz F.L. Reis, Ludwig Institute for Cancer Research and Hospital do Câncer A.C. Camargo, Rua Prof. Antonio Prudente 109, 4th floor, São Paulo, SP, Brazil, 01509-010. e-mail: lreis@ludwig.org.br

\title{
Counter-selection facilitated plasmid construction by homologous recombination in Saccharomyces cerevisiae
}

\author{
Peter Robert Anderson ${ }^{1}$ and Yousef Haj-Ahmad ${ }^{2}$ \\ ${ }^{1}$ University of Guelph, Guelph and ${ }^{2}$ Brock University, St. Catharines, ON, Canada
}

BioTechniques 35:692-698 (October 2003)

The ability to shuffle and combine separate DNA fragments is one of the most fundamental activities in molecular biology. For this reason, in vitro manipulation of bacterial plasmid DNA using restriction enzymes is a staple technique of virtually every molecular biologist. Unfortunately, the position and availability of unique or rare restriction enzyme recognition sequences typically dictate the entire direction of subcloning strategies.

Recombinational cloning techniques based on homologous recombination in Saccharomyces cerevisiae can be used to circumvent tedious multiple-step plasmid construction strategies without the need for specific restriction sites (1-4). Unfortunately, the time investment and additional workload associated with the isolation and characterization of the desired recombinant plasmid from heterogeneous populations of S. cerevisiae transformants is a limitation of these otherwise valuable techniques.

The screening workload associated with in vivo plasmid construction techniques in S. cerevisiae has been partially abrogated by the use of various counter-selection techniques to isolate yeast transformants containing only recombinant plasmid $(1,3)$. However, these useful systems have, to this point, been limited exclusively to the construction of specific yeast expression vectors or required time-consuming replica plating to distinguish yeast transformants carrying recombinant plasmid. Here we describe a general method for URA3-based counter-selection during recombinational cloning that effectively eliminates the growth of background yeast transformants carrying regenerated acceptor plasmid by selecting for the growth of yeast containing only recombinant plasmid. Positive yeast transformants generated using this technique harbor exclusively the recombinant plasmid of interest without the need for replica plating (Figure 1).

The utility of the URA3-based counter-selection technique was demonstrated during in vivo construction of adenovirus E1-deletion rescue plasmids (used for the production of recombinant adenovirus vectors). The in vitro manipulation of such viral genomes as bacterial plasmids is typically difficult due to their large size and general lack of unique or rare restriction sites within the virus coding regions being manipulated. An adenovirus rescue vector capable of undergoing recombinational 